\title{
Dentalmaps : un outil novateur dans la prise en charge des patients ayant reçu une irradiation cervico-faciale.
}

\section{Hacquard A, Devisse M, Derrien A, Lelièvre J, Lansonneur C, Boisramé-Gastrin S, Bouchekoua M. (UFR Odontologie Service d'Odontologie CHRU Morvan, Brest)}

L'ostéoradionécrose ou ostéonécrose post radique (ORN) est une complication orale majeure de la radiothérapie cervico-faciale. Le risque d'ORN étant corrélé à la dose délivrée à l'os de soutien (Chaux-Bodard et al. 2004), la détermination de la dosimétrie est un enjeu primordial dans la prévention de ces complications.

Avec la modernisation des techniques d'irradiation, la cartographie des zones irradiées s'est grandement modifiée.

En effet, les techniques classiques visaient un champ bidimensionnel, pour lequel la dose était uniforme et donc facilement visualisable par le radiothérapeute, le chirurgien-dentiste et le patient.

Les techniques actuelles, comme la radiothérapie conformationnelle en modulation d'intensité (RCMI) ou intensity modulated radiation therapy (en anglais, IMRT), en pleine évolution, permettent de délivrer une dose définie avec précision sur la zone cible tout en épargnant le plus possible les tissus sains et les organes à risque environnants.

Pour évaluer le risque d'ostéoradionécrose avant tout geste invasif (avulsion dentaire, mise en place chirurgicale d'un implant), la connaissance de la dose reçue dans une zone anatomique donnée est indispensable. C'est la difficulté de la RCMI, car la dose n'est pas homogène dans le volume total irradié.

Actuellement, la documentation de la dose, manuellement, est fastidieuse et imprécise.

La création d'un outil simple et adapté (Thariat et al. 2012) permettant de déterminer, de façon automatique, la dosimétrie zone par zone et de pouvoir en déduire un tableau de risque d'ostéoradionécrose liée à la dose est une grande avancée.

C'est ce que propose le logiciel Dentalmaps (Thariat et al. 2011) créé par des radiothérapeutes français à destination des chirurgiens-dentistes. II s'agit d'un outil informatique de segmentation automatique basée sur un multi-atlas (Ramus et al. 2010) mis au point à partir de données d'imagerie de patients traités pour un cancer cervico-facial.

II s'agit d'un outil simple, précis, rapide et applicable en routine, autant pour les radiothérapeutes que pour les chirurgiens-dentistes.

La prévention des complications de l'irradiation cervico-faciale est une quête que l'on doit mener sans relâche afin de préserver la qualité de vie des patients autant d'un point de vue fonctionnel que social. 